\title{
AXIOMATIC COHOMOLOGY FOR BANACH MODULES
}

\author{
I. G. CRAW
}

\begin{abstract}
A characterisation is given of the cohomology functor for Banach modules over a fixed Banach algebra. The result is used to simplify the proofs of two known isomorphism theorems.
\end{abstract}

1. Introduction. In [2], B. E. Johnson shows how the Hochschild cohomology theory for modules over an algebra may be extended to define (useful) cohomology groups $H^{n}(\mathfrak{A}, \mathfrak{X})$ for Banach modules $\mathfrak{X}$ over an arbitrary Banach algebra $\mathfrak{A}$. These groups are given explicitly in terms of a certain cochain complex. We draw attention in this note to the abstract characterisation of these groups. This characterisation has also been noted by J. L. Taylor, and in [5, Proposition 3.7] he obtains a theorem for more general topological algebras, which includes our Theorem 1. Our main concern with the result here is that it simplifies the proofs of known isomorphism theorems.

If $\mathfrak{A}$ is a Banach algebra, $\Delta(\mathfrak{A})$ is the double centralizer algebra of $\mathfrak{A}$, and $\mathfrak{X}$ is a Banach $\Delta(\mathfrak{A})$-module, then Johnson has shown [2, Proposition 1.9] that, with certain restrictions on the module, $H^{n}\left(\mathfrak{A}, \mathfrak{X}^{*}\right) \cong$ $H^{n}\left(\Delta(\mathfrak{R}), \mathfrak{X}^{*}\right) \quad(n=0,1, \cdots)$. A similar result is due to Sinclair [4]; he shows that if $\mathfrak{J}$ is an ideal in $\mathfrak{A}$ having a bounded approximate identity, and $\mathfrak{X}$ is a Banach $\mathfrak{2} / \mathfrak{J}$-module, then $H^{n}\left(\mathfrak{A}, \mathfrak{X}^{*}\right) \cong H^{n}\left(\mathfrak{H} / \mathfrak{J}, \mathfrak{X}^{*}\right)$. By exploiting the uniqueness involved in the characterisation of these cohomology groups, we are able to give shorter, although nonconstructive, proofs of these two isomorphisms.

We assume familiarity with the definitions and notation used in Chapter 1 of [2]. In particular, we write $L^{n}(\mathfrak{A}, \mathfrak{X})$ for the space of continuous $n$-linear maps from $\mathfrak{A}$ to $\mathfrak{X}$, writing conventionally $L^{\mathfrak{0}}(\mathfrak{R}, \mathfrak{X})=\mathfrak{X}$. If $\mathfrak{X}$ is a Banach $\mathfrak{A}$-module, we define $\delta^{0}=0$, and for $n \geqq 0$,

$$
\delta^{n+1}: L^{n}(\mathfrak{R}, \mathfrak{X}) \rightarrow L^{n+1}(\mathfrak{A}, \mathfrak{X})
$$

Received by the editors January 3, 1972 and, in revised form, June 12, 1972. AMS (MOS) subject classifications (1970). Primary 46H25; Secondary $18 \mathrm{H} 15$. Key words and phrases. Banach module, Banach algebra, cohomology.

(c) American Mathematical Society 1973 
by

$$
\begin{aligned}
\delta^{n+1} T\left(a_{1}, \cdots, a_{n+1}\right) & =a_{1} \cdot T\left(a_{2}, \cdots, a_{n+1}\right) \\
& +\sum_{i=1}^{n}(-1)^{i} T\left(a_{1}, \cdots, a_{i} a_{i+1}, \cdots, a_{n+1}\right) \\
& +(-1)^{n+1} T\left(a_{1}, \cdots, a_{n}\right) \cdot a_{n+1} \quad\left(a_{1}, \cdots, a_{n+1} \in \mathfrak{X}\right) .
\end{aligned}
$$

It follows that $\delta^{n+1} \delta^{n}=0$, and we define the cohomology groups of $\mathfrak{A}$, with coefficients in $\mathfrak{X}$, by

$$
H^{n}(\mathfrak{A}, \mathfrak{X})=\operatorname{Ker} \delta^{n+1} / \operatorname{Im} \delta^{n} .
$$

I am grateful to both B. E. Johnson and A. M. Sinclair for prepublication copies of their work, and also to C. Maclachlan for drawing my attention to abstract results of the type given in Theorem 1 .

2. The axiomatic characterization. Let $\mathfrak{A}$ be a Banach algebra, $\mathfrak{B}_{1}$ and $\mathfrak{B}_{2}$ Banach $\mathfrak{A}$-modules, and $\mathfrak{X}$ an arbitrary Banach space. The Banach space $L^{1}\left(\mathfrak{B}_{1} \hat{\otimes} \mathfrak{B}_{2}, \mathfrak{X}\right)$ becomes a Banach $\mathfrak{A}$-module if we define

$$
a \cdot T\left(b_{1} \otimes b_{2}\right)=T\left(b_{1} a \otimes b_{2}\right), \quad T \cdot a\left(b_{1} \otimes b_{2}\right)=T\left(b_{1} \otimes a b_{2}\right),
$$

and then extend the maps by linearity and continuity to $\mathfrak{B}_{1} \hat{\otimes} \mathfrak{B}_{2}$.

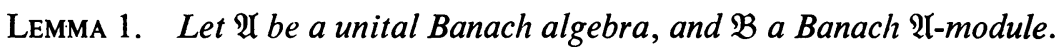
Then for any Banach space $\mathfrak{X}$,

$$
H^{n}\left(\mathfrak{H}, L^{1}(\mathfrak{U} \hat{\otimes} \mathfrak{B}, \mathfrak{X})\right)=\{0\} \quad(n \geqq 1) .
$$

Proof. Let $T \in L^{n}\left(\mathfrak{A}, L^{1}(\mathfrak{U} \hat{\otimes} \mathfrak{B}, \mathfrak{X})\right)$, and suppose that $\delta^{n+1} T=0$; we must find some $S \in L^{n-1}\left(\mathfrak{A}, L^{1}(\mathfrak{U} \hat{\otimes} \mathfrak{B}, \mathfrak{X})\right)$ with $\delta^{n} S=T$. If we define

$$
S\left(a_{1}, \cdots, a_{n-1}\right)(a \otimes b)=T\left(a, a_{1}, \cdots, a_{n-1}\right)(e \otimes b),
$$

then $S$ extends to an element of $L^{n-1}\left(\mathfrak{A}, L^{1}(\mathfrak{A} \hat{\otimes} \mathfrak{B}, \mathfrak{X})\right)$. Now

$$
\begin{aligned}
\delta^{n} S\left(a_{1}, \cdots, a_{n}\right)(a \otimes b)= & T\left(a a_{1}, a_{2}, \cdots, a_{n}\right)(e \otimes b) \\
& +\sum_{i=1}^{n-1}(-1)^{i} T\left(a, a_{1}, \cdots, a_{i} a_{i+1}, \cdots, a_{n}\right)(e \otimes b) \\
& +(-1)^{n} T\left(a, a_{1}, \cdots, a_{n-1}\right)\left(e \otimes a_{n} b\right),
\end{aligned}
$$

and since in particular $\delta^{n+1} T\left(a, a_{1}, \cdots, a_{n}\right)(e \otimes b)=0$,

$$
\begin{aligned}
0= & T\left(a_{1}, \cdots, a_{n}\right)(a \otimes b)-T\left(a a_{1}, \cdots, a_{n}\right)(e \otimes b) \\
& +\sum_{i=1}^{n-1}(-1)^{i+1} T\left(a, a_{1}, \cdots, a_{i} a_{i+1}, \cdots, a_{n}\right)(e \otimes b) \\
& +(-1)^{n+1} T\left(a, a_{1}, \cdots, a_{n-1}\right)\left(e \otimes a_{n} b\right) .
\end{aligned}
$$


Adding, we have

$$
\delta^{n} S\left(a_{1}, \cdots, a_{n}\right)(a \otimes b)=T\left(a_{1}, \cdots, a_{n}\right)(a \otimes b),
$$

and so $\delta^{n} S=T$ as required.

TheOREM 1 (see [3, TheOREM X $\left.3 \cdot 3]^{1}\right)$. Let $\mathfrak{i}$ be a unital Banach algebra, and let $\mathscr{C}$ be the category of unital Banach $\mathfrak{U}$-modules and continuous $\mathfrak{U}$-module homomorphisms. Then for each $n \geqq 0, H^{n}\left(\mathfrak{A},{ }_{-}\right)$is a covariant functor from $\mathscr{C}$ to the category of vector spaces and linear maps such that

(i) $H^{0}(\mathfrak{A}, \mathfrak{X})=\{x \in \mathfrak{X}: x a=a x(a \in \mathfrak{A})\}$ for each object $\mathfrak{X} \in \mathscr{C} ; H^{0}(\mathfrak{U}, \theta)=$ $\theta \mid H^{0}(\mathfrak{A}, \mathfrak{X})$ for each morphism $\theta \in \mathscr{C}$.

(ii) If $0 \rightarrow \mathfrak{X} \rightarrow \mathfrak{Y} \rightarrow \mathfrak{Z} \rightarrow 0$ is an exact sequence in $\mathscr{C}$, split as a sequence of Banach spaces, then there is a long exact sequence

$$
\cdots \rightarrow H^{n}(\mathfrak{U}, \mathfrak{X}) \rightarrow H^{n}(\mathfrak{A}, \mathfrak{Y}) \rightarrow H^{n}(\mathfrak{U}, \mathfrak{3}) \rightarrow H^{n+1}(\mathfrak{A}, \mathfrak{X}) \rightarrow \cdots .
$$

(iii) If $\mathfrak{X}$ is an object in $\mathscr{C}$, so is $L^{1}(\mathfrak{A} \hat{\otimes} \mathfrak{A}, \mathfrak{X})$, and

$$
H^{n}\left(\mathfrak{A}, L^{1}(\mathfrak{A} \otimes \mathfrak{A}, \mathfrak{X})\right)=\{0\} \quad(n \geqq 1) .
$$

Further, if $K^{n}(\mathfrak{H}, \ldots)$ is another sequence of functors having these properties, then there is a natural isomorphism $K^{n}(\mathfrak{A}, \mathfrak{X}) \cong H^{n}(\mathfrak{A}, \mathfrak{X})(n \geqq 0)$.

Proof. We note first that $H^{n}\left(\mathfrak{A},_{-}\right)$has the above properties. It is immediate from the definition that (i) holds, (ii) is proved in [2, Proposition 1.7], while (iii) follows from Lemma 1.

To establish the uniqueness result consider the map $\theta: \mathfrak{X} \rightarrow L^{1}(\mathfrak{A} \hat{\otimes} \mathfrak{A}, \mathfrak{X})$ given by $\theta(x)=T_{x}$, where we define $T_{x}(a \otimes b)=a x b(a, b \in \mathfrak{A})$ and then extend $T$ by linearity and continuity to a map on $\mathfrak{A} \hat{\otimes} \mathfrak{A}$. Since $\mathfrak{A}$ is unital this is an embedding, and has a continuous left inverse given by $T \backsim$ $T(e \otimes e)$. Thus if $\mathfrak{Y}=L^{1}(\mathfrak{A} \hat{\otimes} \mathfrak{U}, \mathfrak{X}) / \theta(\mathfrak{X}), \mathfrak{Y}$ is a Banach $\mathfrak{A}$-module, and

$$
0 \rightarrow \mathfrak{X} \rightarrow L^{1}(\mathfrak{U} \hat{\otimes} \mathfrak{I}, \mathfrak{X}) \rightarrow \mathfrak{Y} \rightarrow 0
$$

satisfies the hypotheses of (ii). Thus using (ii) and (iii) we have

$$
H^{n}(\mathfrak{Y}, \mathfrak{Y}) \cong H^{n+1}(\mathfrak{I}, \mathfrak{X}) \quad(n \geqq 1) .
$$

From (i), $H^{0}\left(\mathfrak{A},{ }_{-}\right)$and $K^{0}\left(\mathfrak{A},{ }_{-}\right)$are naturally equivalent. Using (ii) with the above sequence, and (iii) we have

$$
H^{1}(\mathfrak{A}, \mathfrak{X}) \cong H^{0}(\mathfrak{A}, \mathfrak{Y}) / \operatorname{Im} H^{0}\left(\mathfrak{A}, L^{1}(\mathfrak{A} \hat{\otimes} \mathfrak{A}, \mathfrak{X})\right) \cong K^{1}(\mathfrak{A}, \mathfrak{X}) .
$$

The general result now follows by induction using (1).

\footnotetext{
1 The reader is warned that in the first printing of [3], the condition is misprinted.

$$
H^{n}\left(\Lambda, \operatorname{Hom}_{K}(\Lambda \otimes \Lambda, M)\right)=\{0\} \quad(n>0)
$$
}


RemARKS. Theorem 1 still holds if we replace $\mathscr{C}$ by the category $\mathscr{C}_{1}$ of duals of unital Banach $\mathfrak{A}$-modules, and weak * continuous $\mathfrak{I}$-module homomorphisms. To see this, note first that if $\mathfrak{X}$ is any Banach $\mathfrak{A}$-module, then $L^{1}\left(\mathfrak{H} \hat{\otimes} \mathfrak{A}, \mathfrak{X}^{*}\right) \cong[\mathfrak{H} \hat{\otimes} \mathfrak{U} \hat{\otimes} \mathfrak{X}]^{*}$, while $\mathfrak{I} \hat{\otimes} \mathfrak{A} \hat{\otimes} \mathfrak{X}$ has an obvious $\mathfrak{A}-$ module structure whose dual multiplication is that given on $L^{1}\left(\mathfrak{H} \hat{\otimes} \mathfrak{A}, \mathfrak{X}^{*}\right)$. Thus condition (iii) still holds. Further, since the embedding $\theta: \mathfrak{X}^{*} \rightarrow$ $L^{1}\left(\mathfrak{A} \hat{\otimes} \mathfrak{A}, \mathfrak{X}^{*}\right)$ is weak * continuous, the cokernel of $\theta$ is also a dual Banach $\mathfrak{A}$-module, and so the proof can be carried out within $\mathscr{C}_{1}$.

A similar result holds if $\mathfrak{H}$, although not unital, has a bounded two sided approximate identity. In this case the appropriate category $\mathscr{C}_{2}$ seems to be all duals of neo-unital Banach $\mathfrak{A}$-modules, and weak * continuous $\mathfrak{U}$-module homomorphisms. Recall that an $\mathfrak{A}$-module $\mathfrak{X}$ is neo-unital if, for each $x \in \mathfrak{X}$, there are $a, b \in \mathfrak{A}$ and $y \in \mathfrak{X}$ such that $x=a y b$; equivalently, by the module version of the Cohen factorisation theorem [1, VII 32.22], for each $x \in \mathfrak{X},\left\|e_{\alpha} x-x\right\| \rightarrow 0,\left\|x e_{\alpha}-x\right\| \rightarrow 0$, where $\left\{e_{\alpha}\right\}$ is the bounded approximate identity in $\mathfrak{A}$. This latter condition is easily verified for the module $\mathfrak{A} \hat{\otimes} \mathfrak{U} \hat{\otimes} \mathfrak{X}$, so $L^{1}\left(\mathfrak{Y} \hat{\otimes} \mathfrak{A}, \mathfrak{X}^{*}\right)$ is an object of $\mathscr{C}_{2}$. The next lemma, stated in slightly more generality than we need at present, shows that (iii) of Theorem 1 remains valid in this situation.

LemMA 2. Let $\mathfrak{A}$ be a Banach algebra, and $\mathfrak{J}$ an ideal in $\mathfrak{I}$ containing a bounded approximate identity. Let $\mathfrak{B}$ be a Banach $\mathfrak{A}$-module, and $\mathfrak{X} a$ Banach space. Then

$$
H^{n}\left(\mathfrak{A}, L^{1}\left(\mathfrak{J} \hat{\otimes} \mathfrak{B}, \mathfrak{X}^{*}\right)\right)=\{0\} \quad(n \geqq 1) .
$$

Proof. Let $T \in L^{n}\left(\mathfrak{A}, L^{1}\left(\mathfrak{J} \hat{\otimes} \mathfrak{B}, \mathfrak{X}^{*}\right)\right)$ and let $\left\{e_{\alpha}\right\}$ be the bounded approximate identity in $\mathfrak{I}$. Then $T(\cdot, \cdots, \cdot)\left(e_{\alpha} \otimes \cdot\right)$ is a bounded net in $L^{n}\left(\mathfrak{I}, L^{1}(\mathfrak{B}, \mathfrak{X} *)\right)=[\mathfrak{U} \hat{\otimes} \cdots \hat{\otimes} \mathfrak{A} \hat{\otimes} \mathfrak{B} \hat{\otimes} \mathfrak{X}]^{*}$; hence there is a subnet $\left\{e_{\gamma}\right\}$ such that $T(\cdot, \cdots, \cdot)\left(e_{\gamma} \otimes \cdot\right)$ is convergent in the weak * topology. In particular, given $a_{1}, \cdots, a_{n} \in \mathfrak{A}, b \in \mathfrak{B}$, then $T\left(a_{1}, \cdots, a_{n}\right)\left(e_{\gamma} \otimes x\right)$ is convergent in the weak * topology on $\mathfrak{X}^{*}$ to some member of $\mathfrak{X}^{*}$.

Now suppose $\delta^{n+1} T=0$, and define

$$
S\left(a_{1}, \cdots, a_{n-1}\right)(j \otimes b)=\lim T\left(j, a_{1}, \cdots, a_{n-1}\right)\left(e_{\gamma} \otimes b\right),
$$

where the limit is taken over the directed set corresponding to the subnet $\left\{e_{\gamma}\right\}$. Then by the above $S$ is well defined and extends to an element of $L^{n-1}\left(\mathfrak{A}, L^{1}\left(\mathfrak{J} \hat{\otimes} \mathfrak{B}, \mathfrak{X}^{*}\right)\right)$. The calculation in Lemma 1 now shows, on making obvious changes, that $\delta^{n} S=T$, and the result follows.

COROLlaRY 1. Theorem 1 remains true when $\mathfrak{A}$ is a Banach algebra with a bounded two sided approximate identity provided that the category $\mathscr{C}$ is replaced by the category $\mathscr{C}_{2}$ of all duals of neo-unital Banach $\mathfrak{N}$-modules, and weak * continuous $\mathfrak{2}$-module homomorphisms. 
Proof. It is clear that (i) and (ii) hold, while (iii) follows from Lemma 2 , on putting $\mathfrak{A}=\mathfrak{J}=\mathfrak{B}$. To establish the uniqueness, let $\left\{e_{\alpha}: \alpha \in \mathscr{A}\right\}$ be the approximate identity in $\mathfrak{A}$, let $\mathfrak{X}$ be any neo-unital Banach $\mathfrak{A}$-module, and for brevity write $\mathfrak{Y}=\mathfrak{U} \hat{\otimes} \mathfrak{A} \hat{\otimes} \mathfrak{X}$. Define $\theta: \mathfrak{X}^{*} \rightarrow \mathfrak{Y} *$ by $\theta(f)=T_{f}$ $\left(f \in \mathfrak{X}^{*}\right)$, where $T_{f}(a \otimes b)=a f b(a, b \in \mathfrak{U})$. As before, $T_{f}$ extends by linearity and continuity to a map in $\mathfrak{Y} *$.

If $f, g \in \mathfrak{X}^{*}$, and $T_{f}=T_{g}$, then in particular, for $\alpha, \beta \in \mathscr{A}, x \in \mathfrak{X}$ we have $\left\langle e_{\beta} x e_{\alpha}, f-g\right\rangle=0$. Since $e_{\beta} x e_{\alpha} \rightarrow x$, this shows that $f=g$ and hence $\theta$ is an embedding.

For fixed $\alpha, \beta \in \mathscr{A}$, the map

$$
e_{\alpha} \otimes e_{\beta}: T \rightsquigarrow T\left(e_{\alpha} \otimes e_{\beta}\right) \quad\left(T \in \mathfrak{Y}^{*}\right)
$$

is in $\left.L^{1}(\mathfrak{Y})^{*}, \mathfrak{X}^{*}\right) \cong[\mathfrak{Y} * \hat{\otimes} \mathfrak{X}]^{*}$. As $\alpha, \beta$ vary this gives a bounded net in a dual space, and hence a subnet $\left\{e_{\gamma} \otimes e_{\delta}\right\}$ weak * convergent to some $\left.p \in L^{1}(\mathfrak{Y})^{*}, \mathfrak{X}^{*}\right)$. Further, for $f \in \mathfrak{X}^{*}$,

$$
\begin{aligned}
p T_{f}(x) & =\lim e_{\gamma} \otimes e_{\delta}\left(T_{f}\right)(x) \quad(x \in \mathfrak{X}) \\
& =\lim \left\langle x, e_{\gamma} f e_{\delta}\right\rangle=\lim \left\langle e_{\delta} x e_{\gamma}, f\right\rangle \\
& =\{x, f\} .
\end{aligned}
$$

where the limit in each case is taken over the directed set corresponding to the subnet $\left\{e_{\gamma} \otimes e_{\delta}\right\}$. Thus $p \theta(f)=f$, and $p$ is a left inverse for $\theta$.

Finally, since $\theta: \mathfrak{X}^{*} \rightarrow \mathfrak{Y}^{*}$ is weak $*$ continuous, it is the dual of the $\mathfrak{A}$-module homomorphism $\theta^{*}: \mathfrak{Y} \rightarrow \mathfrak{X}$, and since $\mathfrak{Y}$ is neo-unital, so is $\operatorname{Ker} \theta^{*}$. We then have a split exact sequence $\left.0 \rightarrow \mathfrak{X}^{*} \rightarrow \mathfrak{Y}\right)^{*} \rightarrow\left(\operatorname{Ker} \theta^{*}\right)^{*} \rightarrow 0$ in $\mathscr{C}_{2}$, and the result follows as in Theorem 1.

3. An application to centralizers. Let $\mathfrak{A}$ be a Banach algebra with a bounded approximate identity $\left\{e_{\alpha}\right\}$, and $\mathfrak{X}$ an $\mathfrak{A}$-neo-unital $\mathfrak{A}$-module. We may regard $\mathfrak{A}$ as a closed ideal in $\Delta(\mathfrak{A})$, the algebra of double centralizers, and there is then a unique way of extending the $\mathfrak{U}$-action on $\mathfrak{X}$ to a (unital) $\Delta(\mathfrak{U})$-action; we define

$$
A \cdot x=\lim _{\alpha}\left(A e_{\alpha}\right) \cdot x, \quad x \cdot A=\lim _{\alpha} x \cdot\left(e_{\alpha} A\right) \quad(x \in \mathfrak{X}, A \in \Delta(\mathfrak{U})) .
$$

The following result was proved in [2, Proposition 1.9].

THEOREM 2. Let $\mathfrak{U}$ be a Banach algebra with a bounded approximate identity, and let $\mathfrak{X}$ be a neo-unital $\mathfrak{A}$-module. Then

$$
H^{n}\left(\mathfrak{A}, \mathfrak{X}^{*}\right) \cong H^{n}\left(\Delta(\mathfrak{A}), \mathfrak{X}^{*}\right) .
$$

Proof. We define a sequence of functors on dual neo-unital modules and weak * continuous $\mathfrak{U}$-module homomorphisms by $K^{n}\left(\mathfrak{A}, \mathfrak{X}^{*}\right)=$ $H^{n}\left(\Delta(\mathfrak{A}), \mathfrak{X}^{*}\right), K^{n}(\mathfrak{A}, \theta)=H^{n}(\Delta(\mathfrak{A}), \theta)(n \geqq 0)$. We establish that $K^{n}\left(\mathfrak{A},{ }_{-}\right)$ 
satisfies (i), (ii) and (iii) of Theorem 1 in the category $\mathscr{C}_{2}$; the isomorphism then follows from the uniqueness proved in Corollary 1 .

Clearly $K^{0}\left(\mathfrak{A}, \mathfrak{X}^{*}\right) \subseteq H^{0}\left(\mathfrak{H}, \mathfrak{X}^{*}\right)$. Let $f \in H^{0}\left(\mathfrak{H}, \mathfrak{X}^{*}\right)$, and $x \in \mathfrak{X}$. Then if $A \in \Delta(\mathfrak{U})$, since $\mathfrak{X}$ is neo-unital,

$$
\begin{aligned}
\langle x, A \cdot f-f \cdot A\rangle & =\langle a y b, A \cdot f-f \cdot A\rangle \quad(a, b \in \mathfrak{R}, y \in \mathfrak{X}) \\
& =\langle a y b \cdot A-A \cdot a y b, f\rangle \\
& =\langle y, b A \cdot f \cdot a\rangle-\langle y, b \cdot f \cdot A a\rangle \\
& =0
\end{aligned}
$$

since $f \in H^{0}\left(\mathfrak{A}, \mathfrak{X}^{*}\right)$, and $A$ is a double centralizer. Since this holds for each $x \in \mathfrak{X}, A f=f A$ and (i) follows. The exactness condition (ii) follows immediately from the exactness of $H^{n}(\Delta(\mathfrak{U}), \ldots)$. Finally,

$$
K^{n}\left(\mathfrak{I}, L^{1}\left(\mathfrak{A} \hat{\otimes} \mathfrak{A}, \mathfrak{X}^{*}\right)\right)=H^{n}\left(\Delta(\mathfrak{U}), L^{1}\left(\mathfrak{A} \hat{\otimes} \mathfrak{I}, \mathfrak{X}^{*}\right)\right)=\{0\} \quad(n \geqq 1)
$$

by Lemma 2 , so (iii) holds.

4. An application to annihilator ideals. Let $\mathfrak{A}$ be a Banach algebra containing a closed ideal $\mathfrak{I}$, and $\mathfrak{X}$ a Banach $\mathfrak{A}$-module on which $\mathfrak{J}$ acts trivially; then $\mathfrak{X}$ may also be considered as an $\mathfrak{A} / \mathfrak{I}$-module. The following result was proved directly by Sinclair [4].

THEOREM 3. Let $\mathfrak{A}$ be a Banach algebra, and $\mathfrak{I}$ a closed ideal in $\mathfrak{A}$ containing a bounded approximate identity. Let $\mathfrak{X}$ be any Banach $\mathfrak{U}$-module which is annihilated by $\mathfrak{I}$. Then $H^{n}\left(\mathfrak{A}, \mathfrak{X}^{*}\right) \cong H^{n}\left(\mathfrak{Y} / \mathfrak{J}, \mathfrak{X}^{*}\right)(n \geqq 0)$.

Proof. We assume first that $\mathfrak{I}$ and $\mathfrak{X}$ are unital. If this does not hold, we may adjoin an identity to $\mathfrak{U}$ to obtain $\tilde{\mathfrak{A}}$, and in a natural way $\mathfrak{X}$ becomes a unital $\tilde{\mathfrak{U}}$-module. Since $(\mathfrak{U} / \mathfrak{J})^{\sim} \cong \tilde{\mathfrak{Y}} / \mathfrak{J}$, and since for $n \geqq 0$ we have $H^{n}(\mathfrak{A}, \mathfrak{X}) \cong H^{n}(\tilde{\mathfrak{A}}, \mathfrak{X})[2,1 . \mathrm{d}]$, the general result follows from that obtained in the unital case.

We define a sequence of functors on the category $\mathscr{C}_{1}$ of duals of unital $\mathfrak{U} / \mathfrak{I}$-modules, and weak * continuous $\mathfrak{X} / \mathfrak{I}$-module homomorphisms, by

$$
K^{n}\left(\mathfrak{U} / \mathfrak{J}, \mathfrak{X}^{*}\right)=H^{n}(\mathfrak{U}, \mathfrak{X} *), \quad K^{n}(\mathfrak{U} / \mathfrak{J}, \theta)=H^{n}(\mathfrak{U}, \theta) \quad(n \geqq 0) .
$$

By the remark following Theorem 1, the isomorphism is established if we show that $K^{n}\left(\mathfrak{U} / \mathfrak{I},{ }_{-}\right)$satisfies the conditions (i)-(iii) of Theorem 1 in $\mathscr{C}_{1}$.

Since $\mathfrak{I}$ annihilates $\mathfrak{X}^{*}$,

$$
\begin{aligned}
H^{0}\left(\mathfrak{A}, \mathfrak{X}^{*}\right) & =\left\{f \in \mathfrak{X}^{*}: a f=f a(a \in \mathfrak{U})\right\} \\
& =\left\{f \in \mathfrak{X}^{*}:(a+\mathfrak{I}) f=f(a+\mathfrak{I})(a+\mathfrak{J} \in \mathfrak{U} / \mathfrak{I})\right\} \\
& =H^{0}\left(\mathfrak{U} / \mathfrak{J}, \mathfrak{X}^{*}\right),
\end{aligned}
$$


and (i) holds. The exactness condition follows immediately from the exactness of $H^{n}(\mathfrak{A},-)$, and it remains to check condition (iii).

Let $\mathfrak{Y}=\mathfrak{U} \hat{\otimes} \mathfrak{A} / \mathfrak{J} \hat{\otimes} \mathfrak{X}$ and make $\mathfrak{Y}$ into a left $\mathfrak{I}$-module by defining $j \cdot\left(a \otimes a^{\prime}+\mathfrak{J} \otimes x\right)=j a \otimes a^{\prime}+\mathfrak{J} \otimes x$. Then by [2, Proposition 1.8] we know that $\mathfrak{I} \hat{\otimes} \mathfrak{A} / \mathfrak{J} \hat{\otimes} \mathfrak{X}$ is closed in $\mathfrak{H} \hat{\otimes} \mathfrak{U} / \mathfrak{J} \hat{\otimes} \mathfrak{X}$, and that the sequence

$$
0 \rightarrow(\mathfrak{J} \hat{\otimes} \mathfrak{A} / \mathfrak{J} \hat{\otimes} \mathfrak{X})^{\perp} \rightarrow L^{1}\left(\mathfrak{U} \hat{\otimes} \mathfrak{U} / \mathfrak{J}, \mathfrak{X}^{*}\right) \rightarrow L^{1}\left(\mathfrak{J} \hat{\otimes} \mathfrak{U} / \mathfrak{J}, \mathfrak{X}^{*}\right) \rightarrow 0
$$

is split as a sequence of Banach spaces. Since

$$
(\mathfrak{J} \hat{\otimes} \mathfrak{U} / \mathfrak{J} \hat{\otimes} \mathfrak{X})^{\perp} \cong L^{1}(\mathfrak{U} / \mathfrak{J} \hat{\otimes} \mathfrak{U} / \mathfrak{I}, \mathfrak{X} *),
$$

we have a split exact sequence of Banach spaces

$$
0 \rightarrow L^{1}\left(\mathfrak{U} / \mathfrak{J} \hat{\otimes} \mathfrak{U} / \mathfrak{J}, \mathfrak{X}^{*}\right) \rightarrow L^{1}\left(\mathfrak{U} \hat{\otimes} \mathfrak{A} / \mathfrak{J}, \mathfrak{X}^{*}\right) \rightarrow L^{1}(\mathfrak{I} \hat{\otimes} \mathfrak{U} / \mathfrak{J}, \mathfrak{X} *) \rightarrow 0 .
$$

Giving each space the $\mathfrak{U}$-module structure described before Lemma 1 , the above is an exact sequence of $\mathfrak{A}$-modules, and weak * continuous $\mathfrak{A}$-module homomorphisms. Taking $\mathfrak{B}=\mathfrak{U} / \mathfrak{I}$ in Lemma 1 , and applying (ii) of Theorem 1 , we have for $n \geqq 1$,

$$
H^{n}\left(\mathfrak{A}, L^{1}\left(\mathfrak{U} / \mathfrak{J} \hat{\otimes} \mathfrak{U} / \mathfrak{J}, \mathfrak{X}^{*}\right)\right) \cong H^{n-1}\left(\mathfrak{H}, L^{1}\left(\mathfrak{J} \hat{\otimes} \mathfrak{U} / \mathfrak{I}, \mathfrak{X}^{*}\right)\right)=0 \quad(n \geqq 2)
$$

by Lemma 2 . Let $T \in H^{0}\left(\mathfrak{A}, L^{1}\left(\mathfrak{I} \hat{\otimes} \mathfrak{A} / \mathfrak{I}, \mathfrak{X}^{*}\right)\right)$. Then using the Cohen factorisation theorem [1, VII 32.26],

$$
\begin{aligned}
T(j \otimes a+\mathfrak{I}) & =T\left(j_{2} j_{1} \otimes a+\mathfrak{I}\right)=j_{1} T\left(j_{2} \otimes a+\mathfrak{J}\right) \\
& =T j_{1}\left(j_{2} \otimes a+\mathfrak{J}\right)=T\left(j_{2} \otimes 0+\mathfrak{I}\right)=0 .
\end{aligned}
$$

Thus $H^{1}\left(\mathfrak{A}, L^{1}\left(\mathfrak{A} / \mathfrak{J} \hat{\otimes} \mathfrak{U} / \mathfrak{J}, \mathfrak{X}^{*}\right)\right)=\{0\}$, completing the proof.

\section{REFERENCES}

1. E. Hewitt and K. A. Ross, Abstract harmonic analysis. II: Structure and analysis for compact groups analysis on locally compact Abelian groups, Die Grundlehren der math. Wissenschaften, Band 152, Springer-Verlag, Berlin, 1970. MR 41 \#7378.

2. B. E. Johnson, Cohomology in Banach algebras, Mem. Amer. Math. Soc. No. 127 (1972).

3. S. Mac Lane, Homology, Die Grundlehren der math. Wissenschaften, Band 114, Academic Press, New York; Springer-Verlag, Berlin, 1963. MR 28 \#122.

4. A. M. Sinclair, Annihilator ideals in the cohomology of Banach algebras, Proc. Amer. Math. Soc. 33 (1972), 361-366.

5. J. L. Taylor, Homology and cohomology for topological algebras, Advances in Math. 9 (1972), 137-182.

Department of Mathematics, King's College, Aberdeen, Scotland 